\title{
PATtERn Detection In the ACtivation SpaCE FOR IDENTIFYING SYNTHESIZED CONTENT
}

\author{
Celia Cintas* \\ IBM Research \\ Nairobi, Kenya
}

\author{
Skyler Speakman \\ IBM Research \\ Nairobi, Kenya
}

A PREPRINT

\author{
Edward McFowland III \\ University of Minnesota \\ Minneapolis, MN, USA
}

\author{
Girmaw Abebe Tadesse \\ IBM Research \\ Nairobi, Kenya
}

\author{
Victor Akinwande \\ IBM Research \\ Nairobi, Kenya
}

\author{
Komminist Weldemariam \\ IBM Research \\ Nairobi, Kenya
}

May 28, 2021

\begin{abstract}
Generative Adversarial Networks (GANs) have recently achieved unprecedented success in photorealistic image synthesis from low-dimensional random noise. The ability to synthesize high-quality content at a large scale brings potential risks as the generated samples may lead to misinformation that can create severe social, political, health, and business hazards. We propose SubsetGAN to identify generated content by detecting a subset of anomalous node-activations in the inner layers of pre-trained neural networks. These nodes, as a group, maximize a non-parametric measure of divergence away from the expected distribution of activations created from real data. This enable us to identify synthesised images without prior knowledge of their distribution. SubsetGAN efficiently scores subsets of nodes and returns the group of nodes within the pre-trained classifier that contributed to the maximum score. The classifier can be a general fake classifier trained over samples from multiple sources or the discriminator network from different GANs. Our approach shows consistently higher detection power than existing detection methods across several state-of-the-art GANs (PGGAN, StarGAN, and CycleGAN) and over different proportions of generated content.
\end{abstract}

\section{Introduction}

The accelerated growth of deep learning models for synthetic generation, such as GANs (Goodfellow et al., 2014), has made it possible to create near realistic fake content at a massive scale, generating thousands of samples in seconds. The generation capabilities range from full synthetic faces (Karras et al., 2017, 2020), or partial image modification, such as attribute editing (Choi et al., 2018; Liu et al., 2019) to image style transfer (Zhu et al., 2017a). The generated samples from GANs were reported to be challenging for the human eye to distinguish from real samples ${ }^{2}$ (Karras et al., 2017). With near realistic generated content and high throughput capacity, several high-profile concerns are on the rise in critical areas such as security, ethics, democracy, and intellectual property rights. If the trend continues, the traditional perspective of treating images ("A picture is worth a thousand words") as reliable and trustworthy content may no longer be valid. This will challenge data-driven decision making in societal and commercial activities. Therefore, an effective technique to detect fake (AI-synthesized) content is crucial for platforms that need to verify content from unknown sources. Several methods for detecting counterfeit or synthetic content employ either ad-hoc forensics features or dedicated deep learning architectures to distinguish fake from real content in a given data type and form. These methods rely on labeled samples from different generative sources, data augmentation via replication processes, or specialized deep learning models and training techniques (Hsu et al., 2020; Zhang et al., 2019). In this paper, we propose SubsetGAN that determines whether a given batch of input samples contains a synthesized subset of

\footnotetext{
${ }^{*}$ The paper is under consideration at Pattern Recognition Letters

${ }^{2}$ https://thispersondoesnotexist.com/
} 


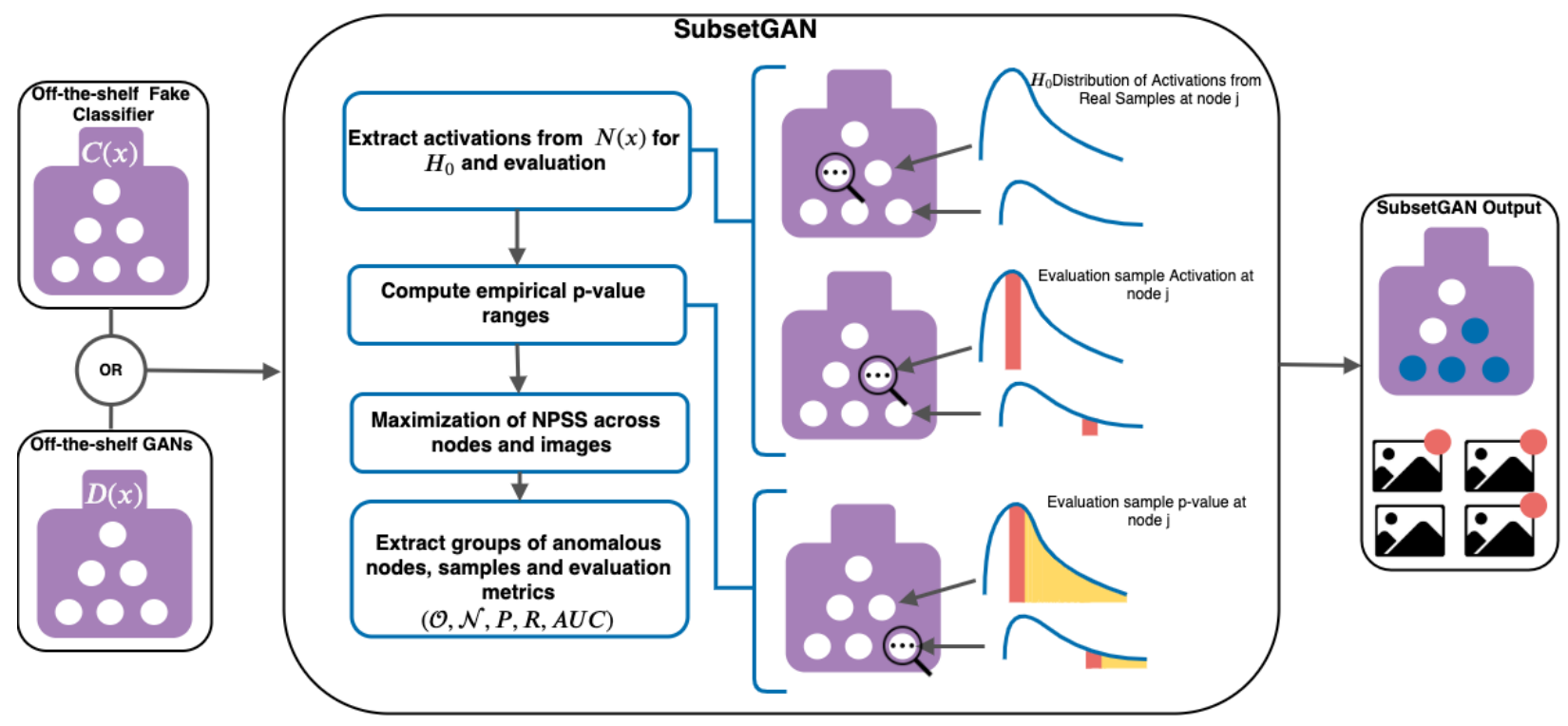

Figure 1: Overview of the proposed approach - SubsetGAN. First, we analyze the distribution of the activation space of the given network. The model can be a pre-trained fake classifier $C(x)$ or the discriminator network of an off-theshelf GAN $D(x)$. After we extracted the activations from the model, we compute the empirical $p$-values followed by the maximization of non-parametric scan statistics - NPSS (Chen and Neill, 2014). Finally, a subset of images and the corresponding anomalous subset of nodes in the network are identified.

samples using an anomalous pattern detection method called group-based subset scanning (Neill, 2012; McFowland III et al., 2013). This work builds on top of recent works that employ individual scanning to detect adversarial attacks across audio and images (Cintas et al., 2020; Akinwande et al., 2020) without exploiting the patterns potentially shared across a group of samples. We hypothesize that synthesized content from off-the-shelf GANs (e.g., PGGAN (Karras et al., 2017), DCGAN (Radford et al., 2015), and StarGAN (Choi et al., 2018)) leave a potentially subtle but systematic trace in the activation space across multiple generated samples. We test this hypothesis through groupbased subset scanning over the activation space that encodes groups of samples that may appear anomalous when analyzed together. In short, this work identifies which, of the exponentially-many, subset of samples in a test set have higher-than-expected activations at which, of the exponentially-many, subset of nodes in a hidden layer of a pre-trained neural network. An overview of the proposed approach is shown in Figure 1.

This work makes four main contributions. First, we show how to detect synthesised image content by applying groupbased subset scanning methods on activations from internal layers of pre-trained models, including binary classifiers (trained to detect fake from real) and state-of-the-art GANs. Second, we present the unique ability to identify patterns of anomalous activations across a group of images. Third, we validated our detection capabilities for both total and partial generation of images from one to multiple generation sources. Fourth, we enhance the performance of the discriminant component of GANs and off-the-shelf fake classifiers to detect the synthesized images without extra labeled examples, data augmentation, or model retraining. We compare the proposed SubsetGAN with state-of-the-art methods, such as FakeSpotter (Wang et al., 2020) and AutoGAN (Zhang et al., 2019). SubsetGAN is also validated across the state-of-the-art generative models (see Tables 2, 4 and 5). We show that the proposed group-based scanning method achieves higher synthesized content detection power compared to existing methods, tested under different types of content generation such as attribute editing, image translation, and full synthetic samples.

\section{Related Work}

Generation of adversarial content can be categorized into two groups: complete (full) and partial. Complete generation is the case where a GAN generates the output from a noise vector (Emami et al., 2018; Han et al., 2018). On the other hand, partial generation is a case where only a part of the content is generated or modified. Examples of partial generation include image translation, and attribute editing. Image translation refers to taking images from one domain and transforming them to have the style of images from another domain. Lastly, attribute editing refers to a case where only the input sample is modified in a specific way for a given characteristic. Next, we review existing methods related to adversarial content generation and the capability of recent techniques to detect them. 


\subsection{Generative Adversarials Networks (GANs)}

GANs have shown remarkable results in various computer vision tasks such as image generation (Emami et al., 2018; Han et al., 2018), image translation (Zhu et al., 2017b; Armanious et al., 2020), face image synthesis (Karras et al., 2017; Ye et al., 2019; Zhang et al., 2018) and recently in generation of text (Chen et al., 2018; Xu et al., 2018) and audio (Lorenzo-Trueba et al., 2018). A typical GAN framework contains generative $(G)$ and discriminative $(D)$ components such that $G$ aims to generate realistic-like content while $D$ learns to discriminate if a generated sample is from the real data distribution $\left(H_{0}\right)$ or not $\left(H_{1}\right)$. Multiple iterations will inform $G$ on how to adjust the generation process to fool $D$ and vice-versa.

We provide a summarised review of the existing methods related to the generation of adversarial content used in this work: DCGAN (Radford et al., 2015), PGGAN (Karras et al., 2017), StarGAN (Choi et al., 2018), and CycleGAN (Zhu et al., 2017a). Deep Convolutional Generative Adversarial Networks (DCGAN) (Radford et al., 2015) is one of the popular and successful networks designed for GAN and commonly used as a standard for image generation . It mainly composes of convolution layers without max pooling or fully connected layers.

More recently, (Karras et al., 2017) presented PGGAN - a new direction for full image synthesis that enhanced the quality of generated images by employing a progressive training approach that fine-tunes layers increasingly over time, and a simple technique to increase variation across the generated images without learnable parameters. Furthermore, PGGAN also provided a novel evaluation technique that takes into consideration both the quality of generated images and their variations.

StarGAN (Choi et al., 2018) is a multiple attribute editing network and its design is aimed at improving the scalability and robustness via a novel GAN that learns the mappings among multiple domains. StarGAN uses only single generator and single discriminator to effectively train a model from images of multiple domains.

Lastly, CycleGAN (Zhu et al., 2017a) is an image translation network that provided a means for image translation in the absence of paired training data, by exploiting the cycle consistent translation property between source and target domains and later combining the disparity from this property with the standard adversarial loss. CycleGAN was validated across different applications, such as collection style transfer, object transfiguration, season transfer and photo enhancement.

\subsection{AI-synthesized Content Detectors}

Existing methods for detecting synthesized content might use forensic-based approaches or deep learning techniques. Several works from these two streams are analyzed below. Raw pixels and ad-hoc forensics features, extracted from real and generated content, were used to train a classifier in (Marra et al., 2018). In (Hsu et al., 2018), the authors proposed a contrastive loss in seeking the typical features of the synthesized images followed by a classifier to detect such AI-generated images. Similarly, (Hsu et al., 2020) reported a deep learning-based approach for identifying the fake images by using the contrastive loss. A simulator was employed to generate images and a spectrum-based classifier was used in (Zhang et al., 2019). Closer to our work, FakeSpotter (Wang et al., 2020) was designed to monitor node behavior to detect generated faces since patterns of layer-by-layer node activation may capture more subtle features that are crucial to detect synthesized content, using a threshold calculated from the average values of outputs in each layer for a given set of training samples.

We hypothesize that more complex layer-by-layer activation behavior underlies learned representations in deep neural networks. Thus, we can enhance average threshold information with a subset of nodes in each layer that yield higher-than-expected activations under generated content. SubsetGAN provides a way to simultaneously detect and characterize multiple samples created by GANs. Unlike the state-of-the-art adversarial content detection techniques, our proposed approach does not require extra labeled examples, data simulation processes, or specialized training techniques, which must be asserted before training time. These capabilities of the proposed approach could also be employed to enhance the performance of existing deep learning methods.

\section{Proposed Approach: SubsetGAN}

Subset scanning treats the detection problem as a search for the most anomalous subset of observations in the data. This exponentially large search space is efficiently explored by exploiting mathematical properties of our measure of anomalousness.

Consider a set of images $X=\left\{X_{1} \cdots X_{M}\right\}$ and nodes $O=\left\{O_{1} \cdots O_{J}\right\}$ within the discriminator $D$ or pre-trained classifier $C$. Let $X_{S} \subseteq X$ and $O_{S} \subseteq O$, we then define the subsets $S$ under consideration to be $S=X_{S} \times O_{S}$. The goal is to find the most anomalous subset: 


$$
S^{*}=\arg \max _{S} F(S)
$$

where the score function $F(S)$ defines the anomalousness of a subset of images and node activations. SubsetGAN uses an iterative ascent procedure that alternates between two steps: a step identifying the most anomalous subset of images $\left(X_{s}\right)$ for a fixed subset of nodes $\left(O_{s}\right)$, or a step that identifies the converse. There are $2^{M}$ possible subsets of images, $X_{S}$, to consider at these steps. However, the Linear-time Subset Scanning property (LTSS) (Neill, 2012; Speakman et al., 2016) reduces this space to only $M$ possible subsets while still guaranteeing that the highest scoring subset will be identified. This drastic reduction in the search space is the key feature that enables SubsetGAN to scale to large networks and sets of images. Without loss of generality, LTSS also decreases the search space of node subsets from $2^{J}$ to $J$ at each of the remaining steps of the ascent procedure. The iterations will converge to a joint, local maximum such that any change to the subset $X_{S}$, conditioned on the subset $O_{S}$, decreases the score $F(S)$. Similarly, any changes to $O_{S}$ conditioned on $X_{S}$ will also decrease $F(S)$. Multiple random restarts are used to approach a global maximum.

\subsection{Non-parametric Scan Statistics (NPSS)}

SubsetGAN uses NPSS that has been used in other pattern detection methods (McFowland III et al., 2013; McFowland et al., 2018; Chen and Neill, 2014). Given that NPSS makes minimal assumptions on the underlying distribution of node activations, SubsetGAN has the ability to scan across different type of layers and activation functions. However, these methods do require baseline or background data to inform their data distribution under the null hypothesis $H_{0}$ of no generated content present.

There are three steps to use non-parametric scan statistics on model's activation data. The first is to form a distribution of "expected" activations at each node. We generate the distribution by letting the discriminator process samples that are known to be real (sometimes referred to as "background" samples) and record the activations at each node. The second step records the activations induced by the group of test images and compares them to the baseline activations created in the first step. This comparison results in a $p$-value at each node, for each image in the test set (Eq. 2). See Figure 2 for an example of this process. Lastly, we quantify the anomalousness of the resulting $p$-values by finding $X_{S}$ and $O_{S}$ that maximize the NPSS, which quantify how much an observed distribution of $p$-values deviates from the uniform distribution. A visual overview of these three steps is shown in Figure 1.

Let $A_{z j}^{H_{0}}$ be the matrix of activations from $Z$ real images at each of $J$ nodes in a discriminator layer. Let $A_{i j}$ be the matrix of activations induced by $M$ images in the test set, that may or may not be generated. SubsetGan computes an empirical $p$-value for each $A_{i j}$, as a measurement for how anomalous the activation value of a potentially generated image $X_{i}$ is at node $O_{j}$. This $p$-value $p_{i j}$ is the proportion of activations from the $Z$ background images, $A_{z j}^{H_{0}}$, that are larger or equal to the activation from an evaluation image at node $O_{j}$.

$$
p_{i j}=\frac{1+\sum_{z=1}^{|Z|} I\left(A_{z j}^{H_{0}} \geq A_{i j}\right)}{|Z|+1}
$$

Where $I(\cdot)$ is the indicator function. A shift is added to the numerator and denominator so that a test activation that is larger than all activations from the background at that node is given a non-zero $p$-value. Any test activation smaller than or tied with the smallest background acivation at that node is given a $p$-value of 1.0.

SubsetGAN processes the matrix of $p$-values $(P)$ from test images with a NPSS to identify a submatrix $S=X_{S} \times O_{S}$ that maximizes $F(S)$, as this is the subset with the most statistical evidence for having been affected by an anomalous pattern. The general form of the NPSS score function is

$$
F(S)=\max _{\alpha} F_{\alpha}(S)=\max _{\alpha} \phi\left(\alpha, N_{\alpha}(S), N(S)\right)
$$

where $N(S)$ is the number of empirical $p$-values contained in subset $S$ and $N_{\alpha}(S)$ is the number of $p$-values less than (significance level) $\alpha$ contained in subset $S$. It has been shown that for a subset $S$ consisting of $N(S)$ empirical $p$-values, $E\left[N_{\alpha}(S)\right]=N(S) \alpha$ (McFowland III et al., 2013). SubsetGAN attempts to find the subset $S$ that shows the most evidence of an observed significance higher than an expected significance, $N_{\alpha}(S)>N(S) \alpha$, for some significance level $\alpha$.

We compare the use of two statistical tests for generated content detection: Berk-Jones (BJ) and Higher-Criticism (HC). BJ test statistic (Berk and Jones, 1979) is defined as:

$$
\phi_{B J}\left(\alpha, N_{\alpha}, N\right)=N * K L\left(\frac{N_{\alpha}}{N}, \alpha\right)
$$


Distribution of Empirical p-values for a test image at a given node
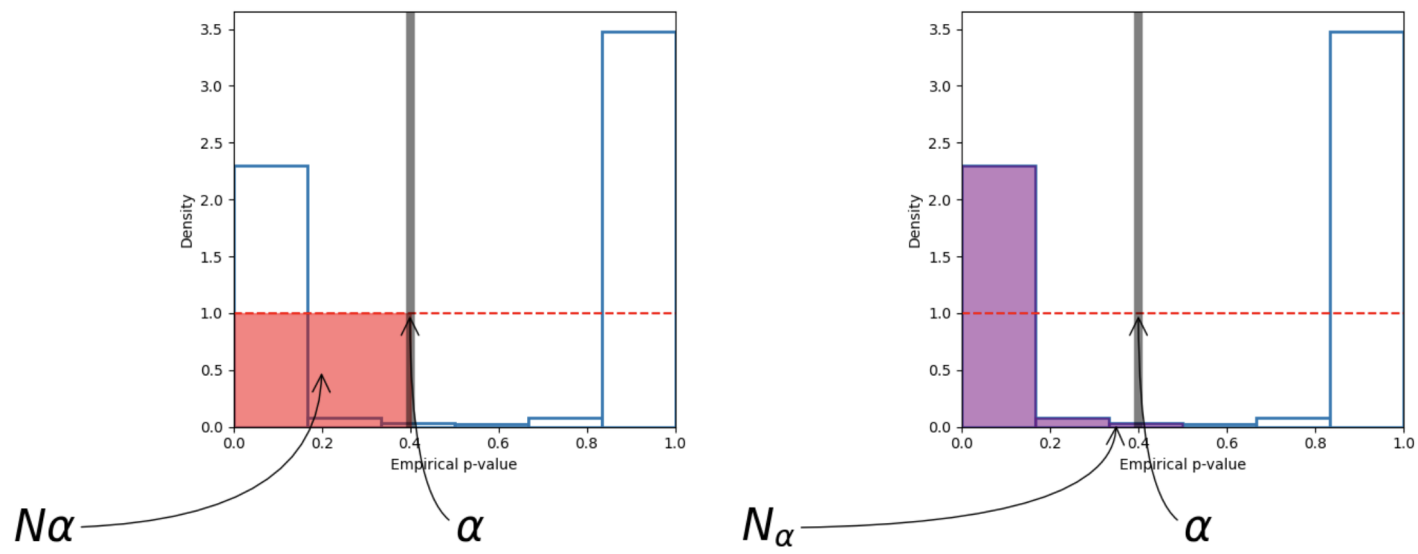

Figure 2: Example of an evaluation image at a given node in order to measure how much the p-values deviate from uniform. Where $N_{\alpha}$ is the number of p-values less than $\alpha, N$ is the number of p-values, and $\alpha$ is the level of significance.

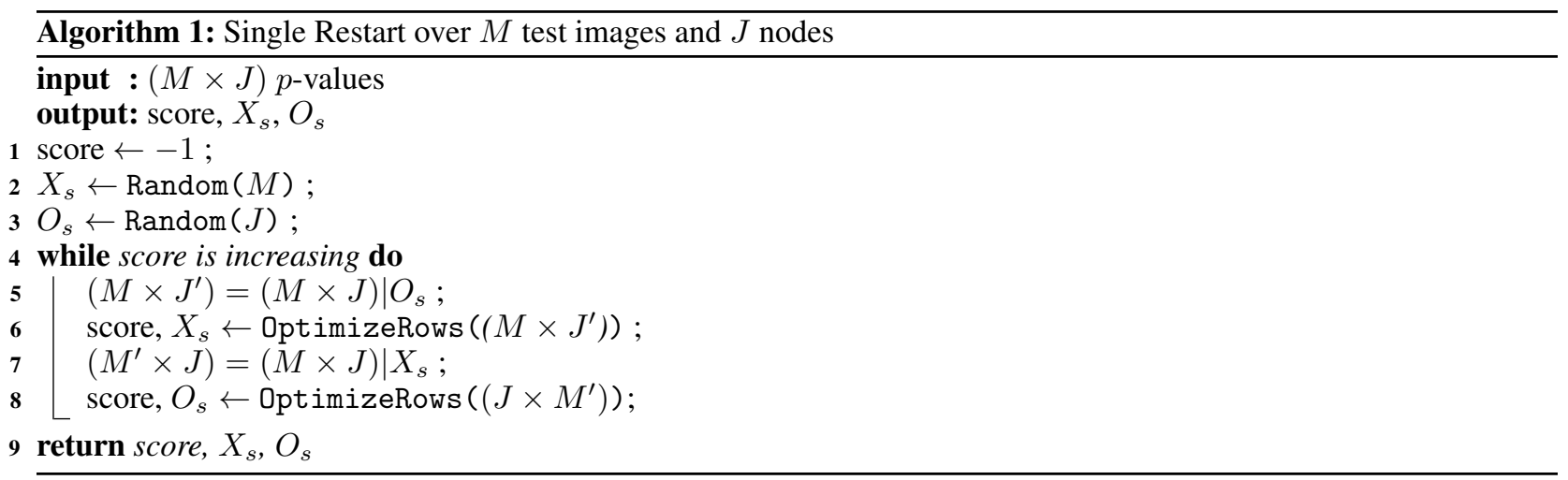

where $K L$ refers to the Kullback-Liebler divergence, $K L(x, y)=x \log \frac{x}{y}+(1-x) \log \frac{1-x}{1-y}$, between the observed and expected proportions of significant $p$-values. We can interpret BJ as the log-likelihood ratio for testing whether the $p$-values are uniformly distributed on $[0,1]$.

The second statistic, Higher-Criticism (Donoho et al., 2004) is defined as

$$
\phi_{H C}\left(\alpha, N_{\alpha}, N\right)=\frac{\left|N_{\alpha}-\alpha N\right|}{\sqrt{N \alpha(1-\alpha)}} .
$$

This can be interpreted as the test statistic of a Wald test for the amount of significant $p$-values given that $N_{\alpha}$ is binomially distributed with parameters $N$ and $\alpha$ under $H_{0}$. Because $\mathrm{HC}$ normalizes by the standard deviation of $N_{\alpha}$, it tends to return small subsets with extreme $p$-values.

\subsection{Efficient maximization of NPSS}

SubsetGAN identifies the anomalous subset of $p$-values through iterative ascent of two optimization steps, see Algorithm 1. Within each step, the number of subsets to consider is reduced from $O\left(2^{E}\right)$ to $O(E)$ where $E$ is the number of elements currently being optimized, either images or nodes, see Algorithm 2. This efficient optimization is a direct application of the LTSS property (Neill, 2012; Speakman et al., 2016). Each element $e$ is sorted by its priority, which is its proportion of $p$-values less than an $\alpha$ threshold. Once sorted, the LTSS property states that the highest-scoring subset will consist of the top- $k$ elements for some $k$ between 1 and $|E|$. Any subset not consisting of the top- $k$ priority elements is sub-optimal and therefore does not need to be evaluated. 


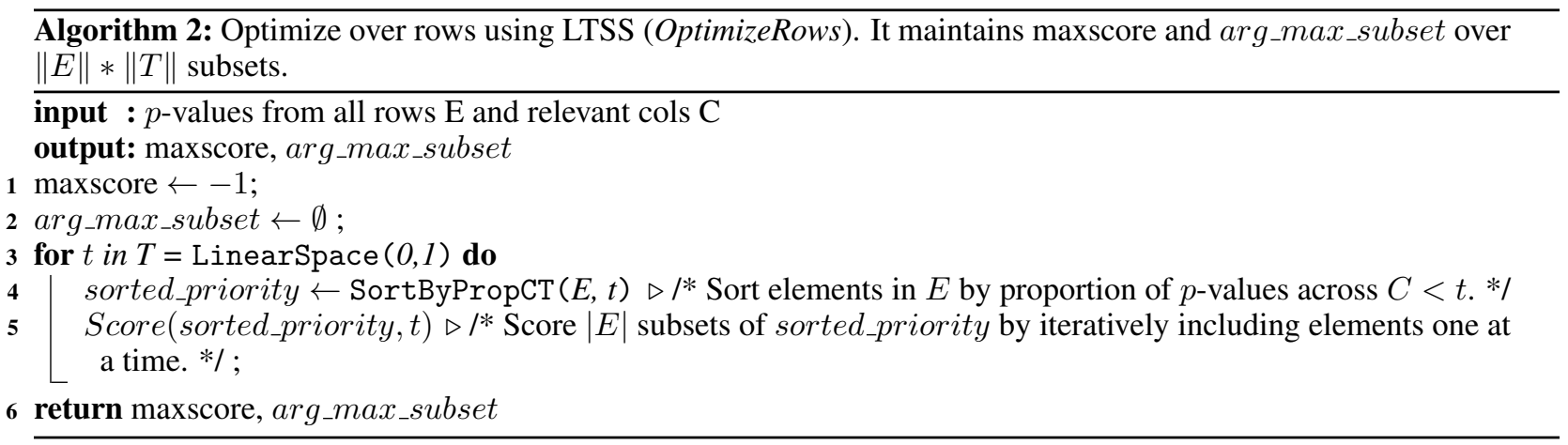

\section{Experimental Setup}

We evaluated the performance of SubsetGAN using multiple score functions, under different experimental scenarios and compared against the state-of-the-art synthesized-content detection methods. The scenarios include different generation types, i.e., complete and partial. Individual-input scanning was used as our baseline. We experimented with the proposed SubsetGAN on the activations drawn from the discriminator components of different pre-trained GANs. SubsetGAN was also validated SubsetGAN on pre-trained classifiers that were trained with samples from multiple GANs (e.g., fake face detectors based on ResNet or SqueezeNet). This helps to provide a scenario that does not require knowledge of the source of the generated content, as is the case for scanning over the discriminator. We also visualized the set of nodes in the discriminators that behaved differently for the anomalous groups. The metrics used are area under receiver operating characteristic curve (AUC), precision $(P)$ and recall $(R)$. In group-scanning results, AUC can be thought of as detection power, which is the method's ability to distinguish between test sets that contain some proportion of synthetic images and test sets containing only real content. P and R reflect detection performance, which is the method's ability to label which images in the test set are synthetic.

\subsection{Datasets, GANs and Methods for Comparison}

For our experiments, we used images from CelebA HQ (Liu et al., 2015), MNIST (LeCun et al., 1998) and CycleGAN datasets (Zhu et al., 2017a) as the background samples. To ensure high-quality and variety of generated samples, we used the pre-trained, highly cited GANs (Section 2) for each of the generation types considered.

To generate completely synthesized samples, we used a pre-trained PGGAN (Karras et al., 2017) model. We selected attribute editing and image translation as use cases for partial generation of samples, and to this end, we used StarGAN (Choi et al., 2018) with two different sets of attributes. The first set contains five attributes (Black Hair, Blond Hair, Brown Hair, Male, and Young). The second set contains only the Brown Hair attribute as proposed by (Wang et al., 2020), for a fair comparison with other methods. For image translation, we used horse2zebra model and its dataset as defined in (Zhu et al., 2017a). Moreover, we also evaluated SubsetGAN over classic DCGAN using benchmarking datasets, such as MNIST.

SubsetGAN scanned over the activations extracted from the discriminator components of the following pre-trained GAN models: DCGAN, CycleGAN ${ }^{3}$, PGGAN $^{4}$ and StarGAN ${ }^{5}$. Furthermore, to present a more general scenario similar to (Wang et al., 2020), we scanned over the activations from two available universal pre-trained fake classifier based on Resnet18 (He et al., 2016) and SqueezeNet (Iandola et al., 2016) architecture and weights. We retrained the model with partial and complete generated samples with accuracy over test samples of 0.912 and 0.920 respectively.In order to compare the synthesized-content detection power of SubsetGAN, we used recently published works: FakeSpotter (Wang et al., 2020), specially designed for fake face detection, and AutoGAN (Zhang et al., 2019), designed for the detection of generic image manipulations.

\subsection{Subset scanning setup}

We run individual and group-based scanning on node activations extracted from several types of layers, which include PGGAN's Scale, GroupScaleZero and Decision layers in addition to common layers such as Conv2D and Batch-

\footnotetext{
${ }^{3}$ https://modelzoo.co/model/pytorch-cyclegan-and-pix2pix

${ }^{4}$ https://pytorch.org/hub/facebookresearch_pytorch-gan-zoo_pgan/

${ }^{5}$ https://modelzoo.co/model/stargan
} 
Table 1: Detection Power (AUC) for group-based and individual subset scanning with two different score functions: BJ and HC, experimented over all layers of DCGAN's discriminator, under two different proportions for MNIST dataset.

\begin{tabular}{lccc|ccc}
\hline \multicolumn{1}{c}{ Layers } & \multicolumn{4}{c}{ group-based and indiv. Subset scan } \\
\hline & $50 \%$ & $30 \%$ & Indv. & \multicolumn{3}{c}{ Score Func. HC } \\
& $30 \%$ & Indv. \\
\hline Conv2d_1 & 1.0 & 1.0 & 1.0 & 1.0 & 1.0 & 1.0 \\
LReLU_1 & 1.0 & 1.0 & 1.0 & 1.0 & 1.0 & 1.0 \\
Conv2d_2 & 1.0 & 0.781 & 0.956 & 1.0 & 0.758 & 0.661 \\
BN2d_1 & 1.0 & 0.803 & 0.956 & 1.0 & 0.778 & 0.661 \\
LReLU_2 & 1.0 & 0.795 & 0.956 & 1.0 & 0.781 & 0.661 \\
Conv2d_3 & 0.378 & 0.383 & 0.528 & 0.487 & 0.531 & 0.523 \\
BN2d_2 & 0.408 & 0.414 & 0.528 & 0.545 & 0.508 & 0.523 \\
LReLU_3 & 0.380 & 0.425 & 0.528 & 0.503 & 0.455 & 0.523 \\
Conv2d_4 & 0.062 & 0.191 & 0.380 & 0.284 & 0.422 & 0.380 \\
Sigmoid & 0.059 & 0.185 & 0.380 & 0.298 & 0.373 & 0.380 \\
\hline
\end{tabular}
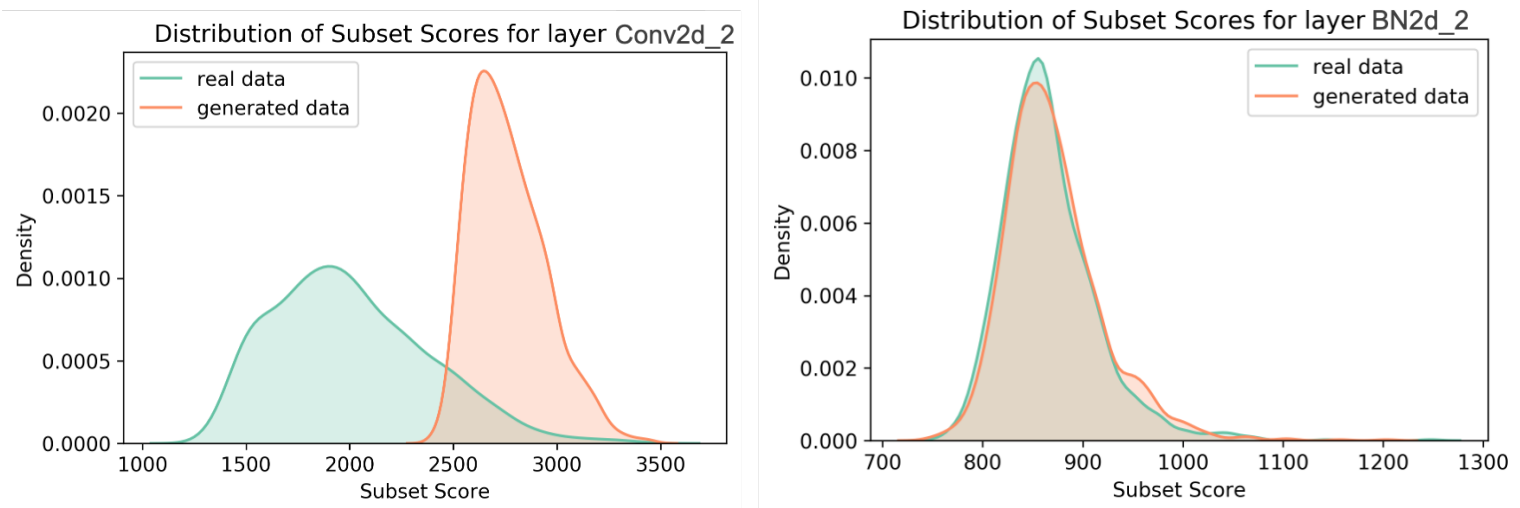

Figure 3: Subset scores distribution across layers of DCGAN $D(x)$ with BJ score function for real and generated samples. The distributions of individual subset scanning scores are shown in green for real images (expected distribution), and in orange for generated samples. Higher AUCs are expected when distributions are separated from each other (See layer Conv2d_2) and lower AUCs when they overlap (See BN2d_2). The computed AUC for the subset score distributions can be found in Table 1 .

Table 2: Comparison, in Precision (P) and Recall (R), of the proposed and existing adversarial detection methods under different Generation Types (GT): TS: total synthesis, AE: attribute editing.

$$
\text { Detection methods }
$$

SubsetGAN FakeSpotter (Wang et al., 2020) AutoGAN (Zhang et al., 2019)

\begin{tabular}{ccc|cc|cc}
\hline GT & P & R & P & R & P & R \\
\hline TS (PGGAN) & 0.941 & 0.900 & $\mathbf{0 . 9 8 6}$ & $\mathbf{0 . 9 8 7}$ & 0.926 & 0.974 \\
AE (StarGAN) & $\mathbf{0 . 9 9 8}$ & $\mathbf{0 . 9 9 9}$ & 0.901 & 0.865 & 0.690 & 0.567 \\
\hline
\end{tabular}

Norm2D. We run group-based scanning across several proportions of generated content in a group, ranging from $10 \%$ to $50 \%$. We used $Z=5000$ images to obtain the background activation distribution $\left(A^{H_{0}}\right)$ for experiments regarding PGGAN, StarGAN and DCGAN,. For evaluation, each test set had images drawn from a larger set of 1000 real images (separate from Z) and from 1500 generated samples. The proportion represented in a test set varied in our experiments. For CycleGAN, we followed the same proportion with the sample size from the horsezzebra dataset (Zhu et al., 2017a). 


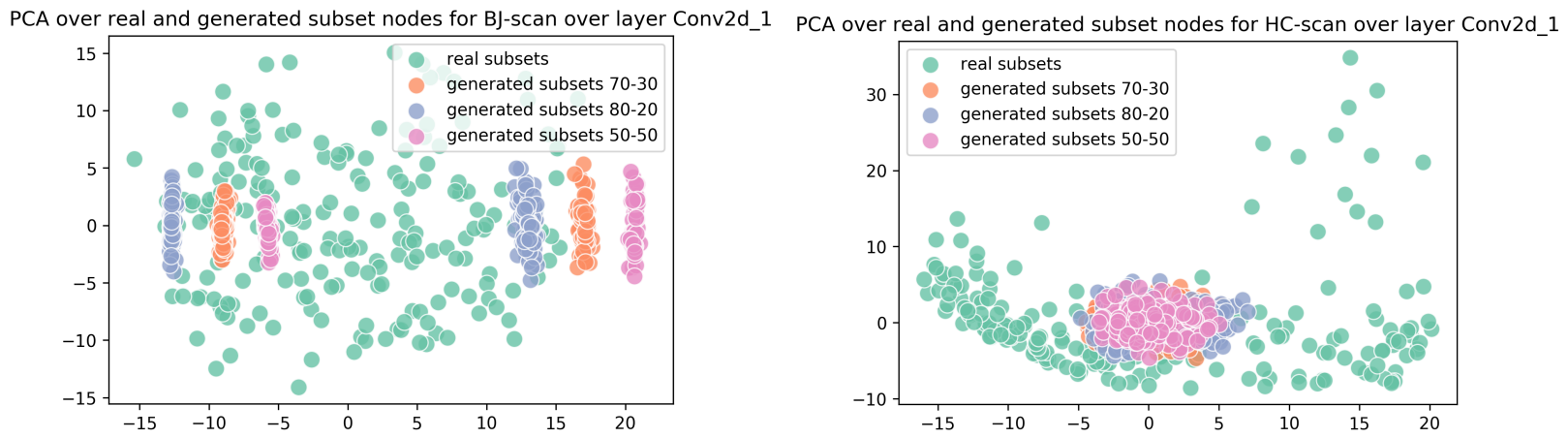

Figure 4: PCA for subsets of nodes for two different score functions BJ and HC from DCGAN over MNIST dataset. In Conv2d_1, we can observe distinctive anomalous subsets of nodes, as we have perfect AUCs at that layer (See Table 1).

Table 3: Detection Power for various detection methods with multiple network settings for different generation types: TS: total synthesis, AE: attribute editting.

\begin{tabular}{cccc}
\hline Method & Generation Type & Network & AUC \\
\hline FakeSpotter & TS & Fake face classifier & 0.985 \\
FakeSpotter & AE & Fake face classifier & 0.881 \\
\hline AutoGAN & TS & GAN & 0.948 \\
AutoGAN & AE & GAN & 0.656 \\
\hline SubsetGAN (indv) & TS & $D(x)$ from PGGAN & 0.950 \\
SubsetGAN (indv) & AE & $D(x)$ from StarGAN & 0.999 \\
\hline SubsetGAN (group) & TS & $D(x)$ from PGGAN & 0.999 \\
SubsetGAN (group) & AE & $D(x)$ from StarGAN & 1. \\
SubsetGAN (group) & AE \& TS & Fake classifier (ResNet) & 0.941 \\
SubsetGAN (group) & AE \& TS & Fake classifier (SqueezeNet) & 0.994 \\
\hline
\end{tabular}

\section{Results}

We compared detection power of two different non-parametric measures of anomalousness: Berk-Jones (BJ) and Higher Criticism (HC) (see Section 3) for MNIST data generated by DCGAN. Table 1 shows AUC for both measures and two proportions (50\% and 30\%) of synthetic content in each test set. Berk-Jones provides better detection power particularly when scanning over individual images only. Scanning over earlier layers in the discriminator provides better detection power than deeper layers. An example of subset scores distributions for this network can be seen in Fig 3.

To better understand the subsets of anomalous nodes that were identified by SubsetGAN, we used dimensionality reduction techniques to visualise which subsets were similar-to or distinct-from each other. We performed principal component analysis on the vector representations of the subsets of nodes. Figure 4, shows each subset of nodes returned by SubsetGAN across the top- 2 principal components. We observe that $\mathrm{HC}$ returns one consistent group of nodes when synthetic inputs are in the test set for both proportions while in BJ varies across ratios but with similar patterns. Furthermore, we observe the randomness of the anomalous nodes when the test set contains all real images. Under this condition, SubsetGAN does not identify any consistent group of nodes with higher activations. The rest of our experiments in this paper are all executed with the BJ score function to measure anomalousness. Below, we provide the results achieved by SubsetGAN and existing methods in detecting partially, and completely synthesized samples. We have also evaluated the proposed SubsetGAN across different generation types, discriminator networks and universal fake classifiers as shown in Table 3. 

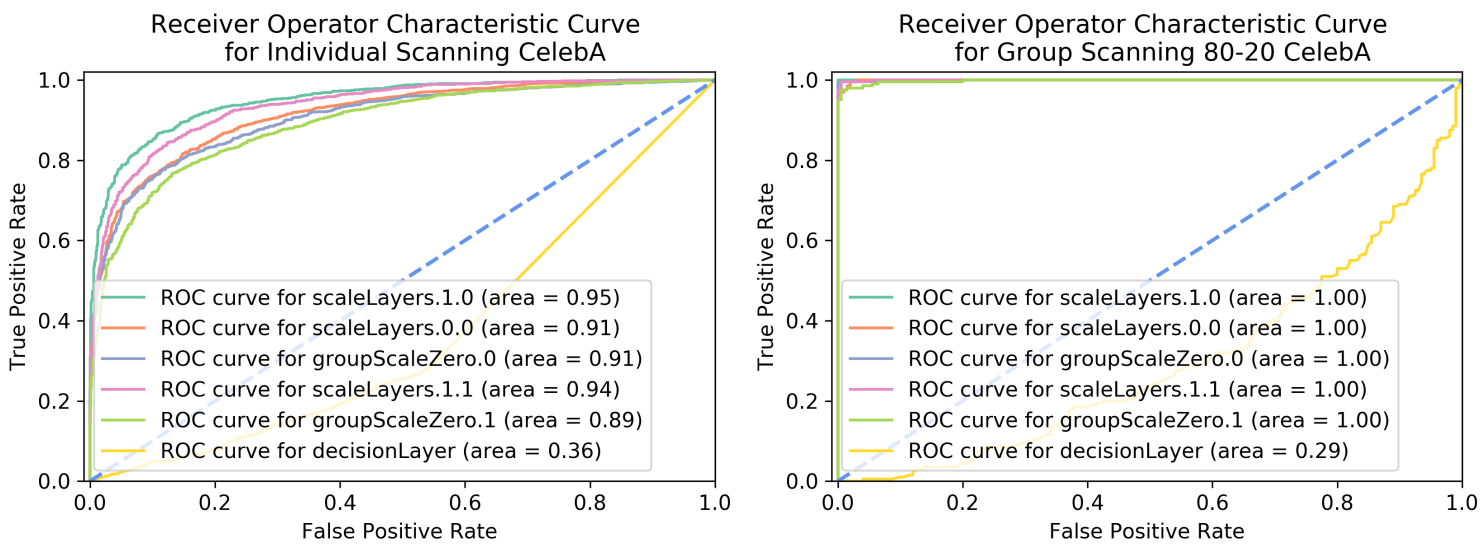

Figure 5: ROC curves for individual and group scanning over 80\%-20\% proportion for PGGAN with CelebA-HQ.

Table 4: Detection power (in AUC) for group-based and individual subset scanning over all layers of StarGAN $D(x)$ under two different proportions (50\% and 20\%) for detection of edited attributes that include Black Hair, Blond Hair, Brown Hair, Male and Young (Choi et al., 2018).

\begin{tabular}{lccc}
\hline & \multicolumn{3}{c}{ Scanning } \\
\cline { 2 - 4 } \multicolumn{1}{c}{ Layers } & Group & 20\% & Indv. \\
\hline Conv2d_1 & 1.0 & 0.478 & 0.225 \\
LeakyReLU_1 & 1.0 & 0.694 & 0.225 \\
Conv2d_2 & 1.0 & 0.997 & 0.351 \\
LeakyReLU_2 & 1.0 & 0.995 & 0.351 \\
Conv2d_3 & 1.0 & 1.0 & 0.961 \\
LeakyReLU_3 & 1.0 & 1.0 & 0.961 \\
Conv2d_4 & 1.0 & 1.0 & 0.992 \\
LeakyReLU_4 & 1.0 & 1.0 & 0.992 \\
Conv2d_5 & 1.0 & 1.0 & 0.997 \\
LeakyReLU_5 & 1.0 & 1.0 & 0.997 \\
Conv2d_6 & 1.0 & 0.888 & 0.258 \\
LeakyReLU_6 & 1.0 & 0.922 & 0.258 \\
\hline
\end{tabular}

Table 5: Precision and recall metrics for group-based scanning over different fake content proportions with 200 runs across $D(x)$ PGGAN layers and CelebA-HQ dataset.

\begin{tabular}{lllllll}
\hline layers/prop. & metric & $\mathbf{5 0 \%}$ & $\mathbf{4 0 \%}$ & $\mathbf{3 0 \%}$ & $\mathbf{2 0 \%}$ & $\mathbf{1 0 \%}$ \\
\hline \multirow{2}{*}{ scaleLayers.0.0 } & precision & $0.929 \pm 0.035$ & $0.895 \pm 0.052$ & $0.832 \pm 0.076$ & $0.719 \pm 0.125$ & $0.405 \pm 0.153$ \\
scaleLayers.1.0 & recall & $0.855 \pm 0.051$ & $0.851 \pm 0.058$ & $0.867 \pm 0.072$ & $0.875 \pm 0.071$ & $0.886 \pm 0.105$ \\
& precision & $0.941 \pm 0.034$ & $0.909 \pm 0.046$ & $0.859 \pm 0.066$ & $0.759 \pm 0.110$ & $0.483 \pm 0.162$ \\
scaleLayers.1.1 & precision & $0.900 \pm 0.045$ & $0.900 \pm 0.052$ & $0.904 \pm 0.053$ & $0.912 \pm 0.063$ & $0.938 \pm 0.071$ \\
& recall & $0.885 \pm 0.046$ & $0.899 \pm 0.045$ & $0.841 \pm 0.072$ & $0.727 \pm 0.105$ & $0.416 \pm 0.148$ \\
groupScaleZero.0 & precision & $0.942 \pm 0.037$ & $0.907 \pm 0.048$ & $0.891 \pm 0.058$ & $0.891 \pm 0.058$ & $0.899 \pm 0.068$ \\
& recall & $0.828 \pm 0.054$ & $0.837 \pm 0.059$ & $0.853 \pm 0.071$ & $0.752 \pm 0.108$ & $0.482 \pm 0.108$ \\
groupScaleZero.1 & precision & $0.942 \pm 0.035$ & $0.917 \pm 0.048$ & $0.868 \pm 0.066$ & $0.857 \pm 0.081$ & $0.871 \pm 0.121$ \\
& recall & $0.832 \pm 0.055$ & $0.836 \pm 0.065$ & $0.843 \pm 0.062$ & $0.845 \pm 0.079$ & $0.474 \pm 0.162$ \\
decisionLayer & precision & $0.326 \pm 0.065$ & $0.245 \pm 0.065$ & $0.171 \pm 0.061$ & $0.110 \pm 0.045$ & $0.048 \pm 0.033$ \\
& recall & $0.218 \pm 0.072$ & $0.213 \pm 0.082$ & $0.209 \pm 0.089$ & $0.205 \pm 0.093$ & $0.196 \pm 0.142$ \\
\hline
\end{tabular}




\subsection{Detection of partially synthesized samples}

Attribute editing is considered as an example for partially synthesized content. We compared the performance of SubsetGAN (group-based and individual) and selected existing methods, FakeSpotter (Wang et al., 2020) and AutoGAN (Zhang et al., 2019), in detecting samples (from CelebA-HQ datset) with an edited attribute.

Tables 2 and 3 show that the proposed group-based SubsetGAN outperformed FakeSpotter and AutoGAN across both GAN types (PGGAN and StarGAN). Particularly, both FakeSpotter and AutoGAN struggled to detect partially synthesized contents drawn from StarGAN. Comparatively, FakeSpotter performed higher than AutoGAN, achieving AUC values second to the proposed SubsetGAN that gains its detection power by efficiently identifying a subset of nodes that deviate away from expected behavior. Other methods may rely on aggregate changes (i.e. a change in average across all node activations). This ability to identify a group of nodes maintains high detection power when only part of the image is edited. Furthermore, SubsetGAN (group) shows that the identified anomalous set of nodes persists across multiple edited samples, which underlines it unique ability to identify patterns of anomalous activations across a group of images.

We also evaluated SubsetGAN across different layers of StarGAN, with more edited attributes and different ratios of synthesized samples in a group. Both individual and group-based scanning resulted in impressive detection performance, particularly in the middle layers of StarGAN as shown in Table 4. Moreover, SubsetGAN achieved impressive detection performance when the ratio of synthesized samples is $50 \%$. Generally, SubsetGAN exhibited higher detection of samples even when only a few attributes are edited. Similar experiments were performed for an other validation task, i.e. image translation. Specifically, we tested SubsetGAN with CycleGAN dataset horse2zebra (Zhu et al., 2017a), yielding 0.973 AUC from individual scan, 0.997 from group-based scanning with $P=0.990$ and $R=0.996$.

\subsection{Detection of completely synthesized samples}

In addition to our validation on detection of partially generated samples, we also evaluated SubsetGAN in detecting completely synthesized samples. We used synthesized samples from PGGAN, real samples from CelebA-HQ dataset and group-based SubsetGAN was applied to detect those synthesized samples. The detection performance across different layers of PGGAN and for different ratios of synthesized samples are shown in Table 5. We started with a $50 \%$ proportion because it is a standard procedure to use minibatch discrimination that looks at multiple examples (real and generated) rather than in isolation, as this helps avoid a collapse of the generator (Salimans et al., 2016).

Results showed that the intermediate layers had more discriminative activations compared to the other layers, consistent with partially synthesized-content detection using StarGAN (see Table 4). Compared to other synthesized samples ratios, $50 \%$ achieved superior trade-off between precision and recall, as expected, given the more balanced positive and negative samples in the group. Smaller ratios, e.g., $10 \%$, exhibited higher recall values due to the lower likelihood of samples being predicted as false negatives. This is validated by the low precision values achieved by smaller ratios due to the higher likelihood of samples being predicted as false positives.

Figure 5 shows the ROC curves with their corresponding AUROC across different layers of the discriminator for CelebA-HQ real samples and completely synthesized samples from PGGAN, across several proportions from groupbased scan and individual scan. Table 5 shows precision and recall averaged across 200 runs for group-based scanning over different fake content proportions over the discriminator layers of PGGAN and using CelebA-HQ dataset. We observe across different experiments with DCGAN and PGGAN, that the first layers maintain high detection power (AUC) when the proportion is higher than $20 \%$ of generated samples, but decays when the amount of generated samples is below or equal to $10 \%$. In this case, opting for individual scan will yield better detection power.

\subsection{Run-time benchmark}

Scalability is often an issue in most existing anomalous sample detection techniques, particularly, when the group of samples is large. After all, there are exponentially many subsets with respect to the group size. To this end, SubsetGAN utilises linear-time subset scanning property that helps to scan across samples in linear time via its ranking function. In Table 6 we can see the execution time for subset scanning under a convolutional layer (main.2) with 131, 200 learnable parameters from PGGAN $D(x)$. For the evaluation we performed 200 runs with the Berk-Jones (Berk and Jones, 1979) score function. We evaluated the proposed method for single and multiple images (each image is $256 \times 256$ pixels) as input for the network. The tests were performed in a desktop machine (2.9 GHz Quad-Core Intel Core i7, 16 GB 2133 MHz LPDDR3). 
Table 6: Benchmark for SubsetGAN. Scan time involves p-value calculation and scanning process for both evaluation samples. Total time measure the complete pipeline from node activation extraction till output metrics recording.

\begin{tabular}{ccc}
\hline \# images & Scan time (secs) & Total time (secs) \\
\hline 1 & $62.0 \pm 0.43$ & $67.7 \pm 0.49$ \\
10 & $77.7 \pm 0.41$ & $83.8 \pm 0.82$ \\
100 & $94.41 \pm 0.42$ & $100.7 \pm 0.94$ \\
1000 & $248.5 \pm 1.27$ & $262.77 \pm 1.34$ \\
\hline
\end{tabular}

\section{Conclusion and Future Work}

We proposed SubsetGAN - a novel method to detect AI-synthesized content via subset scanning. Unlike SubsetGAN, existing methods to detect synthesized content often require labelled generated examples, re-training of models and/or augmentation of data. Our proposed method works by analysing the activation space of the discriminator component of any given generative network or off-the-shelf fake classifier. SubsetGAN provides both the subset of the input images identified as AI-synthesized and the corresponding nodes in the network that gave rise to the identification of those images. With this approach, we aim to enhance the detection power of current deep learning based fake detectors.

SubsetGAN (individual) can operate on a per-image basis and shows strong detection power without requiring multiple images in a test set. This is because our method does not require aggregate-level changes to the activation space to detect generated content. However, additional unique insights are gained with the ability to identify anomaolaous nodes across a group of images. We validated SubsetGAN across different generative networks (e.g., PGGAN, StarGAN, CycleGAN and DCGAN) and types of generation: partial (e.g., attribute editing) and complete. Further, we evaluated the case where no information of the generative source is provided, with general fake classifiers, that are trained with samples from multiple GANs and generation types. We compared the detection capability of SubsetGAN with existing methods FakeSpotter and AutoGAN. The results showed that SubsetGAN outperformed those existing methods consistently across different validation scenarios, and also drives towards interpretability of the detection process. Future work includes utilising the characteristics of the anomalous nodes in improving the generative component. SubsetGAN also sets the foundation to transition from robust detection of generated content to explainability and retraining of generative models.

\section{References}

Victor Akinwande, Celia Cintas, Skyler Speakman, and Srihari Sridharan. 2020. Identifying Audio Adversarial Examples via Anomalous Pattern Detection. In Workshop on Adversarial Learning Methods for Machine Learning and Data Mining, KDD'20.

Karim Armanious, Chenming Jiang, Marc Fischer, Thomas Küstner, Tobias Hepp, Konstantin Nikolaou, Sergios Gatidis, and Bin Yang. 2020. MedGAN: Medical image translation using GANs. Computerized Medical Imaging and Graphics 79 (2020), 101684.

Robert H. Berk and Douglas. H. Jones. 1979. Goodness-of-fit test statistics that dominate the Kolmogorov statistics. Zeitschrift fär Wahrscheinlichkeitstheorie und Verwandte Gebiete 47 (1979), 47-59.

Feng Chen and Daniel B. Neill. 2014. Non-parametric Scan Statistics for Event Detection and Forecasting in Heterogeneous Social Media Graphs. In KDD ’14. 1166-1175.

Liqun Chen, Shuyang Dai, Chenyang Tao, Haichao Zhang, Zhe Gan, Dinghan Shen, Yizhe Zhang, Guoyin Wang, Ruiyi Zhang, and Lawrence Carin. 2018. Adversarial text generation via feature-mover's distance. In NIPS. 46664677.

Yunjey Choi, Minje Choi, Munyoung Kim, Jung-Woo Ha, Sunghun Kim, and Jaegul Choo. 2018. StarGAN: Unified Generative Adversarial Networks for Multi-Domain Image-to-Image Translation. In IEEE CVPR.

Celia Cintas, Skyler Speakman, Victor Akinwande, William Ogallo, Komminist Weldemariam, Srihari Sridharan, and Edward McFowland. 2020. Detecting Adversarial Attacks via Subset Scanning of Autoencoder Activations and Reconstruction Error. In IJCAI 2020.

David Donoho, Jiashun Jin, et al. 2004. Higher criticism for detecting sparse heterogeneous mixtures. The Annals of Statistics 32, 3 (2004), 962-994.

Hajar Emami, Ming Dong, Siamak P Nejad-Davarani, and Carri K Glide-Hurst. 2018. Generating synthetic CTs from magnetic resonance images using generative adversarial networks. Medical physics 45, 8 (2018), 3627-3636. 
Ian J. Goodfellow, Jean Pouget-Abadie, Mehdi Mirza, Bing Xu, David Warde-Farley, Sherjil Ozair, Aaron Courville, and Yoshua Bengio. 2014. Generative Adversarial Networks. (2014), 1-9. https://doi.org/10.1001/ jamainternmed.2016.8245 arXiv:1406.2661

Changhee Han, Hideaki Hayashi, Leonardo Rundo, Ryosuke Araki, Wataru Shimoda, Shinichi Muramatsu, Yujiro Furukawa, Giancarlo Mauri, and Hideki Nakayama. 2018. GAN-based synthetic brain MR image generation. In 2018 IEEE 15th International Symposium on Biomedical Imaging (ISBI 2018). 734-738.

Kaiming He, Xiangyu Zhang, Shaoqing Ren, and Jian Sun. 2016. Deep residual learning for image recognition. In Proceedings of the IEEE conference on computer vision and pattern recognition. 770-778.

Chih-Chung Hsu, Chia-Yen Lee, and Yi-Xiu Zhuang. 2018. Learning to detect fake face images in the wild. In 2018 International Symposium on Computer, Consumer and Control (IS3C). IEEE, 388-391.

Chih-Chung Hsu, Yi-Xiu Zhuang, and Chia-Yen Lee. 2020. Deep fake image detection based on pairwise learning. Applied Sciences 10, 1 (2020), 370.

Forrest N Iandola, Song Han, Matthew W Moskewicz, Khalid Ashraf, William J Dally, and Kurt Keutzer. 2016. SqueezeNet: AlexNet-level accuracy with 50x fewer parameters and $0.5 \mathrm{MB}$ model size. arXiv preprint arXiv:1602.07360 (2016).

Tero Karras, Timo Aila, Samuli Laine, and Jaakko Lehtinen. 2017. Progressive growing of gans for improved quality, stability, and variation. arXiv preprint arXiv:1710.10196 (2017).

Tero Karras, Samuli Laine, Miika Aittala, Janne Hellsten, Jaakko Lehtinen, and Timo Aila. 2020. Analyzing and improving the image quality of stylegan. In Proceedings of the IEEE/CVF Conference on Computer Vision and Pattern Recognition. 8110-8119.

Yann LeCun, Léon Bottou, Yoshua Bengio, and Patrick Haffner. 1998. Gradient-based learning applied to document recognition. Proc. IEEE 86, 11 (1998), 2278-2324.

Ming Liu, Yukang Ding, Min Xia, Xiao Liu, Errui Ding, Wangmeng Zuo, and Shilei Wen. 2019. Stgan: A unified selective transfer network for arbitrary image attribute editing. In CVPR. 3673-3682.

Ziwei Liu, Ping Luo, Xiaogang Wang, and Xiaoou Tang. 2015. Deep Learning Face Attributes in the Wild. In Proceedings of International Conference on Computer Vision (ICCV).

Jaime Lorenzo-Trueba, Fuming Fang, Xin Wang, Isao Echizen, Junichi Yamagishi, and Tomi Kinnunen. 2018. Can we steal your vocal identity from the Internet?: Initial investigation of cloning Obama's voice using GAN, WaveNet and low-quality found data. arXiv preprint arXiv:1803.00860 (2018).

Francesco Marra, Diego Gragnaniello, Davide Cozzolino, and Luisa Verdoliva. 2018. Detection of gan-generated fake images over social networks. In 2018 IEEE Conference on Multimedia Information Processing and Retrieval (MIPR). IEEE, 384-389.

E. McFowland, III, S. Somanchi, and D. B. Neill. 2018. Efficient Discovery of Heterogeneous Treatment Effects in Randomized Experiments via Anomalous Pattern Detection. ArXiv e-prints (March 2018). arXiv:stat.ME/1803.09159

Edward McFowland III, Skyler D Speakman, and Daniel B Neill. 2013. Fast generalized subset scan for anomalous pattern detection. The Journal of Machine Learning Research 14, 1 (Jun 2013), 1533-1561.

Daniel B. Neill. 2012. Fast subset scan for spatial pattern detection. Journal of the Royal Statistical Society (Series B: Statistical Methodology) 74, 2 (2012), 337-360.

Alec Radford, Luke Metz, and Soumith Chintala. 2015. Unsupervised representation learning with deep convolutional generative adversarial networks. arXiv preprint arXiv:1511.06434 (2015).

Tim Salimans, Ian Goodfellow, Wojciech Zaremba, Vicki Cheung, Alec Radford, and Xi Chen. 2016. Improved techniques for training gans. In NIPS. 2234-2242.

Skyler Speakman, Sriram Somanchi, Edward McFowland III, and Daniel B. Neill. 2016. Penalized Fast Subset Scanning. Journal of Computational and Graphical Statistics 25, 2 (2016), 382-404. https://doi .org/10. 1080/10618600.2015.1029578 arXiv:https://doi.org/10.1080/10618600.2015.1029578

Run Wang, Felix Juefei-Xu, Lei Ma, Xiaofei Xie, Yihao Huang, Jian Wang, and Yang Liu. 2020. FakeSpotter: A Simple yet Robust Baseline for Spotting AI-Synthesized Fake Faces. (2020).

Jingjing Xu, Xuancheng Ren, Junyang Lin, and Xu Sun. 2018. Diversity-promoting gan: A cross-entropy based generative adversarial network for diversified text generation. In Proceedings of the 2018 Conference on Empirical Methods in Natural Language Processing. 3940-3949. 
Linbin Ye, Bob Zhang, Meng Yang, and Wei Lian. 2019. Triple-translation GAN with multi-layer sparse representation for face image synthesis. Neurocomputing 358 (2019), 294-308.

Han Zhang, Tao Xu, Hongsheng Li, Shaoting Zhang, Xiaogang Wang, Xiaolei Huang, and Dimitris N Metaxas. 2018. Stackgan++: Realistic image synthesis with stacked generative adversarial networks. IEEE transactions on pattern analysis and machine intelligence 41, 8 (2018), 1947-1962.

Xu Zhang, Svebor Karaman, and Shih-Fu Chang. 2019. Detecting and simulating artifacts in gan fake images. In 2019 IEEE International Workshop on Information Forensics and Security (WIFS). IEEE, 1-6.

Jun-Yan Zhu, Taesung Park, Phillip Isola, and Alexei A Efros. 2017a. Unpaired Image-to-Image Translation using Cycle-Consistent Adversarial Networks. In ICCV, 2017 IEEE.

Jun-Yan Zhu, Richard Zhang, Deepak Pathak, Trevor Darrell, Alexei A Efros, Oliver Wang, and Eli Shechtman. 2017b. Toward multimodal image-to-image translation. In Advances in neural information processing systems. 465-476.

\section{A Supplementary Material}

\section{A.1 Additional Experiments on baseline DCGAN and MNIST}

The architecture of the scanned $D(x)$ from DCGAN can be found in Table 7. For a qualitative inspection of the samples used in the experiments, we can observe a PCA overlapping both generated and real samples can be seen in Figure 6.

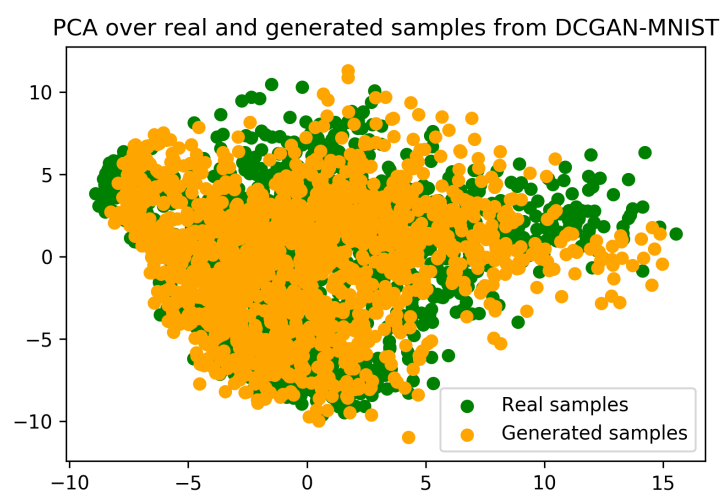

Figure 6: PCA over clean and generated samples from DCGAN-MNIST. To verify that the distributions overlap.
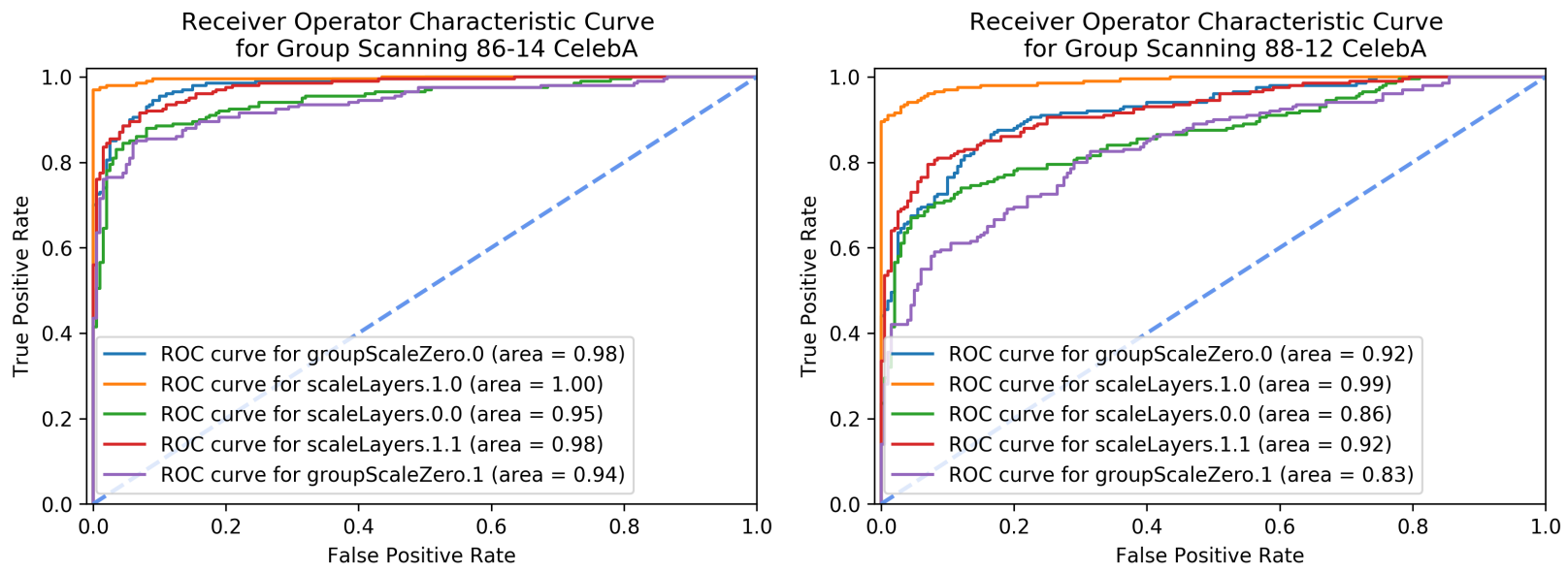

Figure 7: ROC curves for group-based scanning over multiple proportions till 90-10 proportion for PGGAN with CelebA-HQ. 
Table 7: $D(x)$ layers and parameters description from DCGAN

\begin{tabular}{|c|c|c|}
\hline Name & type & parameters \\
\hline main.0 & Conv2d & $\begin{array}{l}\text { kernel_size }=4 \times 4, \text { stride }=2 \times \\
2\end{array}$ \\
\hline main.1 & LeakyReLU & negative_slope $=0.2$ \\
\hline main.2 & Conv2d & $\begin{array}{l}\text { kernel_size }=4 \times 4 \text {, stride }=2 \times \\
2\end{array}$ \\
\hline main.3 & BatchNorm2d & $\begin{array}{l}\text { eps }=1 \mathrm{e}-05 \text {, momentum }=0.1 \text {, } \\
\text { affine }=\text { True }\end{array}$ \\
\hline main.4 & LeakyReLU & \\
\hline main. 5 & Conv2d & $\begin{array}{l}\text { kernel_size }=4 \times 4 \text {, stride }=2 \times \\
2\end{array}$ \\
\hline main.6 & BatchNorm2d & $\begin{array}{l}\text { eps }=1 \mathrm{e}-05, \text { momentum }=0.1 \text {, } \\
\text { affine }=\text { True }\end{array}$ \\
\hline main.7 & Leaky & negative_slope $=0.2$ \\
\hline & Conv2d & $\begin{array}{l}\text { kernel_size }=4 \times 4 \text {, stride }=2 \times \\
2\end{array}$ \\
\hline main.9 & Sigmoid & - \\
\hline
\end{tabular}

\section{A.2 Additional experiments on completely synthesized samples detection}

In Figure 7 we can observe a more detail behaviour for completely synthesized samples across smaller proportions.

\section{A.3 Additional experiments on partially synthesized samples detection}

Regarding image translation with horse2zebra dataset (Zhu et al., 2017a) we report Precision and Recall across layers of CycleGAN $D(x)$ in Table 8.

Table 8: Precision and Recall for group-based subset scanning over all layers of CycleGAN $D(x)$ for image translation with horse2zebra dataset (Zhu et al., 2017a).

\begin{tabular}{lcc}
\hline \multicolumn{1}{c}{ Layers } & \multicolumn{2}{c}{ Group-based Subset scan for $D(x)$} \\
& \multicolumn{2}{c}{$20 \%$} \\
\hline & $\mathrm{P}$ & $\mathrm{R}$ \\
\hline Conv2d_1 & $0.959 \pm 0.022$ & $0.998 \pm 0.007$ \\
LeakyReLU_1 & $0.961 \pm 0.023$ & $0.997 \pm 0.010$ \\
Conv2d_2 & $0.990 \pm 0.006$ & $0.997 \pm 0.010$ \\
InstanceNorm2d_1 & $0.860 \pm 0.041$ & $0.983 \pm 0.025$ \\
LeakyReLU_2 & $0.849 \pm 0.036$ & $0.978 \pm 0.026$ \\
Conv2d_3 & $0.990 \pm 0.005$ & $0.958 \pm 0.036$ \\
InstanceNorm2d_2 & $0.948 \pm 0.026$ & $0.998 \pm 0.007$ \\
LeakyReLU_3 & $0.953 \pm 0.027$ & $0.997 \pm 0.010$ \\
Conv2d_5 & $0.997 \pm 0.011$ & $0.684 \pm 0.099$ \\
InstanceNorm2d_3 & $0.804 \pm 0.080$ & $0.882 \pm 0.055$ \\
LeakyReLU_4 & $0.784 \pm 0.068$ & $0.882 \pm 0.058$ \\
\hline
\end{tabular}

